\title{
IMPORTȦNCIA CLINICA DOS CUSTOS DIRETOS HOSPITALARES EM PACIENTES COM HIPERTENSÃO ARTERIAL EM TRATAMENTO NUM HOSPITAL UNIVERSITÁRIO, RIO DE JANEIRO, BRASIL *
}

\author{
Nelson Albuquerque de Souza e Silva * \\ Guilherme Ribeiro Aguiar * * \\ Armando da Rocha Nogueira *** \\ Marta Maria Turano Duarte ** \\ Regina Helena Fonseca Alves ***
}

\begin{abstract}
SOUZA E SILVA, N.A. de et al. Importância clínica dos custos diretos hospitalares em pacientes com hipertensão arterial em tratamento num hospital universitário, Rio de Janeiro, Brasil. Rev.Saúde públ., S. Paulo, $20: 293-302,1986$.
\end{abstract}

RESUMO: No ano de 1978 foram registrados no Hospital Universitário da Universidade Federal do Rio de Janeiro (HU-UFRJ), 5.262 pacientes moradores da XX Região Administrativa do Rio de Janeiro, Brasil. Quinhentos e três destes prontuários $(9,6 \%)$ com idade $\geqslant 20$ anos foram separados aleatoriamente e 483 destes possuiam registro de pressão arterial (PA) com 138 casos $(28,6 \%$ ) apresentando hipertensão arterial (HA) (PA $\geqslant 140 / 90 \mathrm{mmHg}$ ). Nos 96 hipertensos inicialmente analisados procedeu-se estudos dos custos diretos hospitalares com a avaliação, acompanhamento e tratamento desta população. A PA diastólica inicial destes casos estava assim distribuida: entre 90 e $104 \mathrm{mmHg}-67$ casos $(69,8 \%)$; entre 105 e $114 \mathrm{mmHg}-17$ casos (17,7\%); maior ou igual a $115 \mathrm{mmHg}-12$ casos (12,5\%). 0 período médio de acompanhamento destes grupos foi de 653 dias e somente $1 / 3$ estava com PA controlada ( < 140/90 mmHg) na última consulta. Em 1982, 68,7\% já haviam abandonado tratamento no HUUFRJ. O custo direto total anual por paciente hipertenso em dólares foi de $\$ 102.48$ assim distribuídos: consultas ambulatoriais ligadas à $\mathrm{HA}$ - \$ 33.44; atendimentos de emergência \$ 2.33; internações $\$ 29.92$; exames complementares $\$ 10.45$; despesas com medicamentos anti-hipertensivos $\$ 26.34$. As consultas e internações representam $64 \%$ dos custos e foram em grande parte determinadas pelas complicações da doença. Estas também ocasionam elevados índices de incapacidade temporária e permanente da população de hipertensos com graves repercussōes e custos sociais. A análise dos fatores que contribuem para estes custos indicam a adoção das seguintes medidas para minimizá-los: a) Desenvolvimento de programas que visem melhor controle de HA e menor abandono de tratamento da população já detectada nas próprias unidades do Sistema de Saúde com consequiente redução da morbidade da doença; b) Padronização da avaliação laboratorial e do tratamento com base em estudos de custo-eficácia; c) Hierarquização do sistema de saúde - o hipertenso deve ser tratado em unidades đe saúde onde os custos indiretos hospitalares pouco influenciem os custos das consultas e das internaçōes.

UNTTERMOS: Hipertensão arterial, economia. Hospitais. Custos e análise de custo.

\section{INTRODUÇÃO}

Hipertensão Arterial (HA) é sabidamente doença de alta prevalência na população mesmo em países em desenvolvimen' $c$ como o Brasil 7 ocasionando, quando não tratada adequadamente, complicaçð̃es clínicas graves 3,11 que determinam incapacidade temporária ou mesmo permanente 9 , ocasionando altos custos para $o$ indivíduo e para a sociedade. 0 controle desta doença deve ser considerado como cuidado básico de saúde em todos os países. No entanto, os custos para um programa de detecção, avaliação, tratamento e manutenção em tratamento, da larga população de hipertensos, devem ser cuidadosamente estudados já que os problemas clínicos e sócio-econômicos estão interligados. Uma abordagem inicial do problema de custos na HA envolve a determinação dos custos diretos hospitalares, isto é, o custo das consultas (atendimentos ambulatoriais e de emergência), internações, exames complementares e medicação anti-hipertensiva. Estes estudos podem servir de base para análises econômicas mais complexas e para planejar estratégias prioritárias para o controle da doença.

Em 1982 iniciamos programa de investigação retrospectiva da população de pacientes hipertensos, moradores da XX Região Administrativa do Muni-

* Trabalho realizado com auxílio financeiro parcial do Conselho Nacional de Desenvolvimento Científico e Tecnológ. (CNPq) - Processo no 305194-76. Parte da Dissertação de Mestrado de Guilherme Ribeiro Aguiar, apresentada à Fa culdade de Medicina da UFRJ, em 1984, subordinada ao título "Hipertensão arterial: importância clínica dos custos".

* Da Faculdade de Medicina da Universidade Federal do Rio de Janeiro - Av. Brigadeiro Trompowski s/no - Ilha do Fundão - 21941 - Rio de Janeiro, RJ - Brasil.

** Alunos dos Cursos de Pós-Graduação (mestrado) de Clínica Médica e Cardiologia da Faculdade de Medicina da Universidade Federal do Rio de Janeiro. 
cípio do Rio de Janeiro (Ilha do Governador) e em tratamento no Hospital Universitário da Universidade Federal do Rio de Janeiro (HU.UFRJ), visando adquirir a base inicial de conhecimentos sobre esta população já detectada pelo sistema de saúde para o estabelecimento de programa mais amplo de controle da HA, em toda a comunidade de acordo com os objetivos institucionais do HU-UFRJ *

Neste trabalho estudamos os custos diretos de uma amostra desta população de hipertensos e anali. samos as influências que cada componente formador do custo direto total sofre e as possiveis altemativas para modificá-los em benefício do paciente, da instituição e da sociedade.

\section{MATERIAL E MÉTODOS}

\section{Seleção de Pacientes}

Revimos uma amostra aleatória de 503 prontuá. rios de pacientes com 20 anos de idade ou acima e moradores da XX Região Administrativa do Município do Rio de Janeiro, registrados no HU.UFRJ no ano de 1978, ano da inauguração do Hospital e que permitiria o maior período de acompanhemento possível. Esta amostra representou $9,6 \%$ do total de pacientes da XX Região Administrativa registrados nesse ano no HU.UFRJ (5.262 pacientes).

Selecionamos, inicialmente, os pacientes que tivessem apresentado pelo menos um registro de Pressão Arterial (PA) sistólica $\geqslant 140 \mathrm{mmHg}$ e/ou diastólica $\geqslant 90 \mathrm{mmHg}$ ou com PA abaixo destes limites mas que estivessem em uso de medicação anti-hipertensiva. Vinte prontuários não tinham nenhum registro de PA. Dos 483 restantes, 138 (28,6\%) apresentavam HA primária e $4(0,8 \%) \mathrm{HA}$ secundária. Os 96 casos inciais com HA primária, e que faziam parte dos 138 casos, por nós revistos, foram analisados visando a obter os dados necessários para cálculo dos custos diretos com estes pacientes. Tivemos que encerrar o estudo antes de estudar todos os 138 casos de HA selecionados. Como os 96 casos representam $70 \%$ do total de pacientes selecionados e como em estudo posterior incluindo já os 138 casos encontramos características clínicas idênticas com os 96 casos iniciais, não vimos justificativa para reescrever todos os dados.

\section{Dados Obtidos}

Pressão Arterial - Com a finalidade de classificar os pacientes de acordo com o nível de PA, considerou-se $\circ$ primeiro registro de PA obtido ambulatorialmente embora tivéssemos anotado o valor de todas as PAs registradas durante todo o período de acompanhamento. $O$ valor da PA na última consulta foi utilizado para avaliar o controle dos níveis de PA obtidos com o tratamento.
Consultas Ambulatoriais - Anotamos o número total de consultas médicas ambulatoriais para cada paciente e o número de consultas consideradas como relacionadas à HA. Estas foram definidas como aquelas nas quais o médico adotou alguma atitude em relação à $\mathrm{HA}$.

Consultas de Emergência - Verificou-se o nú. mero de consultas de emergência relacionadas à $\mathrm{HA}$ ocorridas no HU.UFRJ.

Internações - Anotou-se o número de pacientes internados e o número de internações no $\mathrm{HU}$ e a duração das internações ocasionadas por problemas relacionados à $\mathrm{HA}$ ou de internações por problemas não relacionados à HA, nas quais a hipertensão arterial foi fator de prolongamento do período de internação.

Exames Complementares - Verificou-se o número dos seguintes exames complementares realizados em cada paciente:

- Exames de sangue: hemograma, glicemia, uréia, creatinina, ácido úrico, colesterol, triglicerídios, dosagem de sódio e potássio.

- Exames de urina: elementos anormais e sedimento, proteinúria de $24 \mathrm{~h}$, cultura de urina.

- Exames radiológicos: raios $\mathrm{X}$ de tórax, pielografia venosa, arteriografia.

- Eletrocardiograma.

- Outros exames: qualquer outro exame como coronariografia, teste de esforço, renograma, dosagem de ácido vinil mandélico e outros, que pudessem ter relação com a investigação do pa. ciente hipertenso.

Foi impossível determinar com precisão se cada um dos exames acima eram ou não solicitados devido à hipertensão e suas complicações ou devido a outros problemas associados. Por esse motivo utilizamos o número total de exames realizados mesmo que a sua indicação tivesse ocorrido por motivos não relacionados à $\mathrm{HA}$.

Medicamentos - Registrou-se o uso de toda e qualquer droga anti-hipertensiva prescrita para o tra. tamento dos pacientes durante todo o período de acompanhamento anotando-se a dose e o periodo de sua utilização. Considerou-se para cálculo de custo que o paciente fazia uso correto da dose prescrita mesmo se existisse anotação de que o paciente não estava fazendo uso da mesma. Não consideramos o uso de drogas não anti-hipertensivos como digital, anti-arritmicos e outros, mesmo que tivessem sido usados por problemas associados ou conseqüentes à HA.

Periodo de Acompanhamento - Calculou-se o período de acompanhamento ambulatorial de cada

\footnotetext{
- Hospital Universitário da UFRJ. Objetivos institucionais e bases operacionais. 1975. (Documento interno).
} 
paciente através das datas da primeira e da última consulta ambulatorial. Os casos com apenas uma consulta foram excluídos para cálculo do período médio de acompanhamento da população estudada.

Informações sobre custos hospitalares - Todos os dados de custo referem-se ao mês de março de 1983. Nessa ocasião o dólar era cotado a Cr\$ 401,45 e o valor da ORTN * era de Cr\$3.392,32 (US\$ 8.45).

a) Custos das consultas ambulatoriais, de emergência e das internações

O HU.UFRJ tem um centro de custos na Divisão de Finanças para acompanhamento dos custos hospitalares. Os custos das consultas e internações são divididos em custos diretos $e$ indiretos. Os diretos representam as despesas próprias de cada local de prestação de serviço, ou seja, despesas de pessoal, material e despesas gerais (luz, telefone, limpeza). Os indiretos são formados pelo rateio dos centros de custos de base e dos centros de custos intermediários, variando a distribuição destes custos de acordo com a natureza do serviço prestado.

Os custos de base são constituídos pelas atividades de apoio administrativo, enquanto os custos in. termediários são formados pelas atividades de apoio técnico.

São centros de custos de base:

Administração, Serviço de Documentação Médica, Seção de Transporte, Serviço de Material, Centro de Material Esterilizado, Engenharia e Manutenção, Lavanderia e Rouparia, Serviço de Nutrição e Dietética, Serviço de Farmácia, Serviço Social.

\section{São centros de custos intermediários:}

Centro de Tratamento Intensivo, Anestesia, Gasoterapia, Patologia Clínica, Anatomia Patológica, Hemoterapia, Medicina Física e Reabilitação, Radiodiagnóstico, Medicina Nuclear, Postos de Enfermagem (Setores de Internação), Unidades de Métodos Especiais de Diagnóstico, Hemodiálise, Centro Cirúrgico.

0 custo do pessoal médico (professores) é apropriado da seguinte maneira: $50 \%$ do salário é dirigido à atividade assistencial e a outra metade é alocada como despesa não operacional (atividade de ensino e pesquisa). $O$ montante referente a atividade assistencial é distribuído pelas áreas específicas de atuação de cada serviço. No caso da Clínica Médica, por exemplo, $50 \%$ dos gastos com salários dos docentes são divididos pelo Ambulatório, Setores de Internação, Emergência e Centro de Tratamento Intensivo, os quais são os setores de atuação daquela especialidade.
São considerados Setores de Atuação; Ambulatório, Emergência, Setores de Internação, Centro de Tratamento Intensivo, Centro Cirúrgico, Hemodiálise, Unidade de Métodos Especiais.

De modo análogo apura-se os custos dos médicos contratados, inclusive os residentes.

$\mathrm{Na}$ área especifica de atuação do ambulatório, por exemplo, além dos custos diretos próprios, são incluídos todos os itens listados anteriormente dos centros de custos indiretos, exceto a Seção de Transporte e o Serviço de Nutrição e Dietética (Alimentação). Com relação aos centros de custos intermediários entram dados relativos aos serviços de: Anestesia, Patologia Clínica, Anatomia Pa. tológica, Medicina Física e Reabilitação, Radiodiagnóstico, Medicina Nuclear, Unidade de Métodos Especiais, Hemodiálise.

$\mathrm{Na}$ área específica de atuação da Emergência estão computados os custos diretos próprios e um rateio de todos os centros de custos de base mais os dados relativos aos seguintes centros de custos intermediários: Anestesia, Patologia Clínica, Hemoterapia, Radiodiagnóstico, Medicina Nuclear.

$\mathrm{Na}$ área de atuação das clínicas de internação são computados os custos de todos os centros de custos de Base, exceto Transporte e todos os centros de custos intermediários.

\section{b) Custos dos Exames Complementares}

Os custos destes exames são calculados através da Tabela Internacional de Unidades de Serviço (US) a qual leva em consideração o tempo e a đificuldade para realização de cada procedimento e os custos dos materiais e equipamentos utilizados.

O custo total de cada setor ou serviço do Hospital é calculado e dividido pelo número de US realizadas, achando-se assim o valor da US de cada setor. Assim, o custo total do Serviço de Patologia Clínica é o somatório das despesas de pessoal. material, despesas gerais, acrescido do rateio dos centros de custos de base, da Administração, Centro de Material, Engenharia e Manutenção e Serviço de Farmácia.

O custo total do Serviço de Radiologia é, de forma análoga, o somatório das suas despesas diretas mais o rateio dos centros de custos de base, da Administração, Serviço de Material Esterilizado, Manutenção, Rouparia e Farmácia.

O custo total da Unidade de Métodos Especiais é o somatório dos custos diretos próprios e os centros de custos de base, de Administração, Serviço de Documentação Médica, Material, Serviço de Material

* Obrigações Reajustáveis do Tesouro Nacional. 
Esterilizado, Engenharia, Manutenção, Rouparia, Lavanderia e Serviço de Farmácia.

A Tabela 1 mostra o número de Unidades de Serviço de cada exame e seus custos no HU-UFRJ.

TABELA 1

Unidades de Serviço (US) e Custo * dos Exames Complementares - Hospital Universitário da UFRJ (março - 1983)

\begin{tabular}{lcc}
\hline \multicolumn{1}{c}{ Exame } & No de US & Custo $-C_{r} \$$ \\
\hline RX tórax & 12 & $1.147,32$ \\
ECG & 18 & $1.722,06$ \\
Hemograma & 10 & 634,70 \\
Uréia & 05 & 317,35 \\
Creatinina & 07 & 444,29 \\
Acido Úrico & 05 & 317,35 \\
Eletrólitos & 10 & 634,70 \\
Glicemia & 05 & 317,35 \\
Colesterol & 05 & 317,35 \\
Triglicerídios & 10 & 634,70 \\
Urina (EAS) & 05 & 317,35 \\
\hline
\end{tabular}

* Valor da US (março - 1983):

- Radiologia $=\mathrm{Cr} \$ 95,61$

- Métodos Especiais (ECG) $=$ Cr $\$ 95,67$

- Patologia Clínica $=$ Cr $\$ 63,47$

\section{c) Despesas com Medicamentos}

Como não dispúnhamos de dados sobre os custos dos medicamentos anti-hipertensivos utilizamos $o$ preço de venda registrado no Brasindice de março de 1983.

\section{RESULTADOS}

Dos 96 pacientes estudados, 67(70\%) eram do sexo feminino e 29 do sexo masculino, com idade média de 51,5 anos.

\section{1 - Níveis de Pressão Arterial (PA)}

Com base na PA diastólica obtida na primeira consulta no $\mathrm{HU}$, os pacientes foram classificados em 3 grupos:

PAD $90.104 \mathrm{mmHg}-67$ pacientes $(69,8 \%)$.

PAD 105-114 mmHg - 17 pacientes $(17,7 \%)$.

PAD $115 \mathrm{mmHg}-12$ pacientes (12,5\%).

A Tabela 2 mostra o número de pacientes em determinadas faixas de pressões sistólicas e diastólicas ambulatoriais registradas na última consulta (para os 82 pacientes com mais de uma consulta ambulatorial). Como vemos, apenas $32,9 \%$ dos pacientes tiveram sua $\mathrm{PAD}$ reduzida espontaneamente ou com medicação anti-hipertensiva para níveis abaixo de $90 \mathrm{mmHg}$ de diastólica, na última consulta.

\section{2 - Perfodo de Acompanhamento}

Quatorze pacientes tiveram apenas uma consulta ambulatorial, 7 foram acompanhados por menos de 3 meses e o período máximo de acompanhamento foi de 1.772 dias. $O$ periodo médio de
TABELA 2

Nível da Pressão Arterial (PA) na Ultima Consulta Ambulatorial de Pacientes Registrados do Hospital Universitário da UFRJ

\begin{tabular}{cc}
\hline $\begin{array}{c}\text { Nível da PA } \\
(\mathrm{mmHg})\end{array}$ & $\mathrm{n}(\%)$ \\
\hline Sistólica & $29(35,3)$ \\
$<140$ & $24(29,4)$ \\
$140 \cdot 159$ & $29(35,3)$ \\
$\geqslant 160$ & \\
Distólica & $27(32,9)$ \\
$<\quad 90$ & $26(31,7)$ \\
$90 \cdot 99$ & $29(35,4)$ \\
$\geqslant 100$ &
\end{tabular}

PA = pressāo arterial em milímetros de me rcúrio $n(\%)=n$ o de casos e percentual em relação ao total de pacientes com mais de 1 consulta (82 casos)

acompanhamento para os 82 casos com mais de uma consulta ambulatorial foi de 653 dias. Apenas 30 pacientes tiveram acompanhamento contínuo entre 1978 e 1982 . O percentual de $68,7 \%$ dos pacientes abandonaram o tratamento, isto é, não compareceram à consulta há mais de um ano.

3-Consultas Ambulatoriais, Atendimentos na Emergência e Internaçőes:

A Tabela 3 mostra o número de consultas ambu. latoriais. A média de consultas relativas à $\mathrm{HA}$ foi de 4,5 consultas por paciente por ano e representaram $40 \%$ do total de consultas ambulatorias destes pacientes.

TABE LA 3

Número de consultas ambulatoriais e média por paciente com Hipertensāo Arterial/ano segundo o tipo. Hospital Universitánio - UFRJ

\begin{tabular}{lrc}
\hline \multicolumn{1}{c}{$\begin{array}{c}\text { Tipo de } \\
\text { consulta }\end{array}$} & No & Média/Paciente/Ano \\
\hline $\begin{array}{l}\text { Relacionadas à Hipertensão } \\
\text { Arterial }\end{array}$ & 605 & 4,5 \\
Todos os motivos & 1675 & 11,4 \\
\hline
\end{tabular}

Ocorreram 35 atendimentos de emergência no HU para problemas relacionados à $\mathrm{HA}$, ou seja, 0,2 atendimentos de emergência por paciente/ano.

Nove pacientes foram internados no $\mathrm{HU}$ por motivos relacionados à HA (3 por infarto do miocárdio, 3 por acidente vascular cerebral, um por insuficiência cardiaca, um com encefalopatia hipertensiva e um para controle da HA). Estas internações ocuparam 144 dias ou 0,83 dias por paciente/ano.

\section{4 - Custos Diretos das Consultas Ambulatoriais}

No mês de março de 1983 o custo direto (CD) da consulta ambulatorial foi de $\mathrm{Cr} \$ 2.983,00$ (US\$ 7.43) segundo metodologia anteriormente explicada. 
Assim, o custo direto anual das consultas (CDAC) com os pacientes hipertensos foi de: $\mathrm{CDAC}=$ Média de consultas/ano $\times C D$ consultas $=11,4 \times 2.983=$ Cr $\$ 34.006,20 /$ paciente/ano (US\$ 84.70 ).

Com relação apenas ao custo das consultas relativas à $\mathrm{HA}(\mathrm{CDACH})$ temos:

$\mathrm{CDACH}=$ média de consultas/ano $\mathrm{x} C \mathrm{CD}$ consul$\operatorname{tas}=4,5 \times 2.983=\mathrm{Cr} \$ 13.423,50 /$ paciente $/$ ano (US\$ 33.44).

\section{5 -Custos Diretos dos Atendimentos de Emer- gência}

Em março de 1983 o custo direto das consultas de emergência foi de $\operatorname{Cr} \$ 4.680,00$ (US\$ 11,66 ). Sendo assim, o custo direto anual dos atendimentos de emergência (CDAE) dos pacientes hipertensos foi de:

$\operatorname{CDAE}=0,2 \times 4.680=\mathrm{Cr} \$ 936,00 /$ paciente $/$ ano (US\$ 2.33).

\section{6 - Custos Diretos das Internações}

Em março de 1983 o custo dire to das internações foi de $C_{r} \$ 14.472,00 /$ dia de internação (US\$ 36.05 dólares), logo o custo direto anual das internações relativas à $\mathrm{HA}$ (CDAIN) foi de:

CDAIN $=0,83 \times 14.472=\operatorname{Cr} \$ 12.012,00 /$ pacien te/ano (US\$29.92).

\section{7 - Custos Diretos dos Exames Complementares}

$O$ custo foi calculado considerando-se todos os exames realizados que pudessem ter relação com a HA calculando-se em seguida a média de exames por ano e a média anual de cada exame por paciente.

\section{TABELA 4}

Exames Complementares

(número, média/ano e média/paciente/ano) realizados nos pacientes com Hipertensão Arterial) - Hospital Universitário - UFRJ

\begin{tabular}{lrrc}
\hline \multicolumn{1}{c}{ Exame } & Nọ & Média/Ano & Média/Paciente/Ano \\
\hline ECG & 80 & 44,7 & 0,46 \\
RX Tórax & 96 & 53,6 & 0,56 \\
Pielografia & 12 & 6,7 & 0,07 \\
Hemograma & 128 & 71,5 & 0,75 \\
EAS & 149 & 83,2 & 0,87 \\
Eletrólitos & 73 & 40,8 & 0,42 \\
Ácido Urico & 49 & 27,3 & 0,28 \\
Creatinina & 109 & 60,9 & 0,63 \\
Uréia & 123 & 68,7 & 0,71 \\
Glicose & 209 & 116,8 & 1,21 \\
Colesterol & 92 & 51,4 & 0,53 \\
Triglicerídios & 58 & 32,4 & 0,33 \\
\hline
\end{tabular}

A Tabela 4 mostra estes valores para cada exame. No mês de março de 1983 a US do Serviço de Pa. tologia Clínica no HU foi de Cr\$63,47, a do Servi- ço de Radiodiagnóstico de Cr\$ 95,61 e a da Unidade de Métodos Especiais de $\operatorname{Cr} \$ 95,07$. Com estes valores, os dados da Tabela 4 e o número de US de cada exame (Tabela 1) podemos calcular o custo total dos exames/paciente/ano. O valor encontrado foi de Cr $\$ 4.196,00$ (US\$ 10.45).

\section{8-Despesas com Medicamentos}

Tivemos 32 pacientes que não receberam qual. quer droga anti-hipertensiva. Quatro pacientes tinham dados inconclusivos sobre a dose prescrita e 60 casos possuiam dados que permitiam calcular a dose total de cada medicamento prescrito para cada paciente durante todo o periodo de acompanhamento.

A Tabela 5 mostra as drogas utilizadas, a freqüên: cia com que foram prescritos, e seu preço unitário em março de 83 , pelo nome comercial. O gasto anual teórico com as drogas anti-hipertensivas utilizadas nos pacientes tratados (se todas as drogas prescritas tivessem realmente sido consumidas) foi de $\mathrm{Cr} \$$ $846.602,00$ (US\$2,108.86) ou Cr\$14.110,00 (US\$ $35.15)$ por paciente/ano.

\section{TABELA 5}

Medicamentos Utilizados, Frequiência de Uso nos Pacientes com Hipertensão Arterial, e Preço Unitário, Hospital Universitário - UFRJ

\begin{tabular}{|c|c|c|c|}
\hline $\begin{array}{c}\text { Drogas } \\
\text { (Nome Comercial) }\end{array}$ & $\begin{array}{c}\text { No de } \\
\text { Pacientes }\end{array}$ & $\%$ & $\begin{array}{c}\text { Preço de Março } 1983 \\
\text { (em cruzeiros) } \\
\text { por comprimido }\end{array}$ \\
\hline Higroton & 28 & 46,6 & $33,7 \quad(50 \mathrm{mg})$ \\
\hline Aldomet & 25 & 41,6 & $38,26(250 \mathrm{mg})$ \\
\hline Lasix & 17 & 28,3 & 28,2 \\
\hline Drenol & 16 & 26,6 & 9,5 \\
\hline Diclotride & 07 & 11,6 & 12,2 \\
\hline Serpasol & 10 & 16,6 & 7,2 \\
\hline Propranolol & 10 & 16,6 & 12,7 (40mg) \\
\hline Aldazida & 03 & 5,0 & 86,0 \\
\hline Minipress & 02 & 3,5 & $111,1(5 \mathrm{mg})$ \\
\hline Papaverina & 02 & 3,3 & $31,5(0,25 \mathrm{mg})$ \\
\hline Aldactone & 01 & 1,6 & $51,6(25 \mathrm{mg})$ \\
\hline Sotacor & 01 & 1,6 & 79,3 \\
\hline Moduretic & 01 & 1,6 & 29,4 \\
\hline Atenol & 01 & 1,6 & 145,5 \\
\hline Diurana & 01 & 1,6 & 22,2 \\
\hline Raudazida & 01 & 1,6 & 76,1 \\
\hline Pressuren & 01 & 1,6 & $307,7 / \mathrm{ml}$ \\
\hline Hidrion & 01 & 1,6 & 20,2 \\
\hline Dilacoron & 01 & 1,6 & $52,5(80 \mathrm{mg})$ \\
\hline Adelfan & 01 & 1,6 & 9,9 \\
\hline
\end{tabular}

$\%=$ percentagem do total de pacientes tratados com drogas. 
Se incluirmos, para cálculo do gasto anual por paciente, todos os casos, excluindo apenas aqueles que tiveram uma consulta ambulatorial, teremos o valor de Cr $\$ 10.582,00$ por paciente/ano ou US\$ 26.34 .

\section{9 - Custo direto total (CDT) do paciente hiper- tenso no Hospital Universitário (HU)}

Este custo é dado pela fórmula:

$\mathrm{CDT} /$ paciente/ano = custo direto das consultas ambulatoriais + custo direto dos atendimentos de emergência + custo direto das internaçōes + custo direto dos exames complementares + despesas com medicamentos anti-hipertensivos.

$\mathrm{CDT} /$ paciente/ano (em cruzeiros):

$34.006,00+936,00+12.012,00+4.196,00+$ $10.582,00=\operatorname{Cr} \$ 61.732,00$.

CDT/paciente/ano (em dólares):

$84,71+2.33+29.92+10.45+26.36=$ US $\$ 153.77$.

Se considerarmos apenas as consultas ambulatoriais ligadas à HA o CDT/paciente/ano passa a ser de Cr\$ $41.149,00$ ou US\$ 102.48 .

\section{DISCUSSÃO}

As análises de custos em saúde envolvem diversas metodologias e objetivos, desde a simples análise de minimização de custos, isto é, a escolha da melhor de duas alternativas que tenham efeitos idênticos, até as análises mais complexas de custo benefício e custo utilidade que envolvem análise dos resultados a serem obtidos, em termos de qualidade de vida para os pacientes.

O presente estudo enquadra-se mais em uma análise de minimização de custos já que fomece, através de análise retrospectiva de dados registrados, os custos diretos reais de uma população de hipertensos em acompanhamento em um hospital de ensino. A análise subseqüente dos componentes deste custo permite que se proponha estratégias para minimizá-lo, sem afetar a qualidade do atendimento fornecido ao paciente, embora não tenhamos testado na prática a alternativa proposta.

A população de hipertensos estudada distribui-se com relação aos níveis de PA, de modo semelhante a estudos epidemiológicos como o do HDFP3 o qual encontrou $71,5 \%$ de hipertensos com cifras de PAd entre 90 e $104 \mathrm{mmHg}$, percentagem semelhante aos $69,8 \%$ por nós encontrada.

A média encontrada de 4,5 consultas/ano relacionadas à HA é semelhante ao relatado na literatura 12 . Devemos assinalar, no entanto, que notamos grandes variações individuais na freqüência e intervalo de acompanhamento destes pacientes, além do alto indice de abandono de acompanhamento $(68,7 \%)$.
Considerando-se apenas os trinta pacientes que estiveram em acompanhamento contínuo entre 1978 e 1982; a média de consultas anuais relacionadas à HA cai para 3,2 consultas/paciente, e para todas as consultas esta média anual/paciente passa a ser de 7,5 .

O custo das consultas em um hospital de ensino é necessariamente mais elevado devido a necessidade de pessoal especializado, aquisição e manu. tenção de material e equipamentos sofisticados e de altos custos. No entanto, o custo contábil do HU/UFRJ é comparável ao de outros hospitais universitários que adotam sistemas semelhantes na computação de custos como o da Universidade de Santa Maria e o Hospital de Clínicas de Porto Alegre (RS). Assim, segundo documento do Ministério da Educação e Cultura sobre os Hospitais Universitários, no quarto trimestre de $1982^{*}$, os custos diretos das consultas ambulatoriais foram: $\mathrm{Cr} \$ 1.532,42$ no HU.UFRJ, Cr\$ $1.230,10$ em Santa Maria e Cr\$ 2.105,93 em Porto Alegre. Os dados desses hospitais não podem ser comparados ao de outros hospitais do INAMPS (Instituto Nacional de Assistência Médica e Previdência Social), que adotam sistemática diferente para determinação de seus custos.

Devemos ressaltar, no entanto, que o cálculo do custo da consulta ambulatorial, por exemplo, é in. fluenciado pela produtividade e, nesta, foi considerado apenas a produtividade assistencial medida em termos do número de consultas efetivadas. A produtividade de um hospital de ensino não pode ser medida apenas em termos assistenciais, mas deve incluir a formação de recursos humanos e a produção de pesquisa, funções inerentes a seus objetivos. No entanto, não temos ainda metodologia para medir estes itens e incluílos nos cálculos econômicos. A própria produtividade assistencial do serviço de ambulatório do $\mathrm{HU}$ não englobou vários outros serviços efetivamente desempenhados como as consultas dos serviços de enfermagem, de nutrição e do serviço social, realização de curativos, imunizaçōes e testes, programas com grupos de pacientes, além de outros, os quais não são remunerados pelo convênio do HU com o INAMPS. A inclusão de todos estes itens de produtividade certamente reduziria o custo da consulta, significativamente. Assim, por exemplo, no mês de novembro de 1984 o custo total da consulta ambulatorial, considerando como produtividade apenas as consultas médicas, foi de Cr\$ 37.140,00. No entanto, se atribuírmos o mesmo valor da consulta médica aos outros procedimentos e consultas ambulatoriais não incluídos ainda como produtividade, o custo cairia para $\mathrm{Cr} \$$ $28.815,00$, uma redução de $22,4 \%$.

\footnotetext{
* Demonstrativo trimestral de custos hospitalares. Ministério da Educação. Secretaria de Ensino Superior (MEC/SESU). (Documento interno).
} 
A produtividade relativa ao ensino foi de certo modo incluída em nossos cálculos já que atribuímos apenas $50 \%$ do custo do pessoal médico (professores) no cálculo dos custos diretos. No entanto o ensino no ambulatório influi negativamente na produtividade assistencial por aumentar o tempo de consulta por paciente. No HU, a média de consultas/ médico em todos os ambulatórios especializados é de 8,0 por turno de quatro horas, ou seja, meia-hora por paciente (incluindo pacientes de consulta de primeira vez). Consideramos prejudicial à qualidade de atendimento $e$ do ensino tentar aumentar esta média. $O$ custo da consulta não deve ser reduzido aumentando o número de pacientes atendidos, por médico, pois isto afeta a qualidade do atendimento com repercussões adversas para $o$ indivíduo e para a sociedade. Deve-se assinalar, no entanto, que existem grandes variabilidades individuais. Ações sobre médicos com persistente baixa produtividade assistencial poderão reduzir o custo da consulta sem afetar a qualidade de atendimento.

Outro ponto a ressaltar na análise do çusto das consultas é o de que os custos indiretos são duas vezes e meia maiores que os custos diretos dos diversos serviços. Assim, por exemplo, o valor médio da consulta no ano de 1982 foi de Cr $\$ 4.117,00$, entretanto se excluídos os custos indiretos este valor reduzir-se-ia para $C_{I} \$ 1.575,00$ uma redução de $61,7 \%$. Este fato indica que uma doença como a Hipertensão Arterial, que não exige recursos sofisticados para tratamento na grande maioria dos pacientes, não deve ocupar grande número de consultas em hospitais de grande porte e sim ser tratada em unidades de saúde onde o custo indireto não tenha tanta influência no custo da consulta. As unidades de saúde devem adequar sua clientela aos recursos de que dispõem. A racionalidade da hierarquização dos serviços de saúde é em parte suportada pelos dados do presente trabalho.

Com relação às consultas de emergência é possível que a média anual de atendimentos/paciente encontrada não reflita a real necessidade de atendimento emergencial dos nossos pacientes, já que podem ter recorrido a outros postos de urgência. A ação mais eficaz, para reduzir estes custos, estaria mais ao nível de controle adequado da HA para evitar a demanda ao Serviço de Emergência por complicações relativas à doença não controlada, já que sabemos que apenas $32,9 \%$ dos nossos pacientes tiveram PAd reduzida para níveis abaixo de $90 \mathrm{mmHg}$, na última consulta, e sabemos que quanto pior o controle dos níveis de PA mais freqüentes se tornam as suas complicações 3,11 .

Com relação às internações é possível que estas tenham representado as necessidades reais destes pacientes pois a internação em outros hospitais da rede de saúde é mais difícil do que no próprio HU.
A média de 16 dias/internação/paciente é identica à média encontrada para todos os pacientes clínicos no HU. Embora talvez seja possível reduzir esta média com atuações a nível da equipe de saúde de nossas enfermarias, verificamos que as causas destas internações (infarto do miocárdio, acidente vascular encefálico, insuficiência cardiaca) talvez tornem difícil esta redução do tempo de intemação. A ação mais eficaz, em nossa opinião, estaria novamente em obter melhor controle de PA dos pacientes de modo a reduzir a demanda para internaçбes ao evitar-se complicaçōes da doença. Temos conhecimento por outros estudos $3,8,10$ que as complicações hipertensivas (AVC, hipertrofia, ventricular esquerda, cardiomegalia e retinopatia) podem ser reduzidas de até $55 \%$, mesmo na hipertensão leve, embora as complicações ateroscleróticas (infarto do miocárdio, outras doenças coronarianas, entre outras) sejam de redução mais duvidosa com tratamento anti-hipertensivo.

Surpreendentemente para um hospital universitário, geralmente acusados de realizarem exames em excesso, a freqüência de exames complementares solicitados dos pacientes hipertensos foi bem abaixo do que é recomendado por alguns autores 4,5 , mesmo tendo-se considerado todos os exames realizados como teoricamente relacionados à $\mathrm{HA}$, pois foi im. possivel discernir se uma glicemia, por exemplo, era solicitada devido ao diagnóstico de HA ou por outra causa.

A investigação de causas secundárias da $\mathrm{HA}$ foi bastante limitada. A adoção de uma investigação mínima padronizada poderia, no entanto, reduzir mais ainda os custos dos exames complementares. $O$ custo da potassemia, creatinina sérica e EAS, investigação mínima de avaliação inicial e de acompanhamento, do hipertenso sugerida pela "Canadian Hypertension Society"1 seria de apenas Cr $\$ 1.396,00$, bem in ferior ao custo dos exames complementares que encontramos no $\mathrm{HU}$, embora tenhamos de considerar que este custo teórico não inclui exames solicitados por outros motivos, em cada paciente.

$\mathrm{O}$ cálculo dos gastos com medicamentos foi afetado pelo fato de a maioria dos pacientes com HA leve, principalmente com PAd $<100 \mathrm{mmHg}$ não terem sido tratados com medicação anti-hipertensiva ou mesmo diagnosticados como hipertensos. A maioria dos pacientes tratados teve como droga inicial prescrita um diurético tiazídico. No entanto, a droga escolhida foi a Clortalidona (Higroton). Outras vezes, ao invés de tiadízico o diurético inicial era a furosemida mesmo quando não havia indicação especial para esta preferência. Tanto a Clortalidona quanto a Furosemida são drogas bem mais caras que a Hidroclorotiazida, cujo uso mais freqüente pode reduzir os custos com a medicação, principalmente quando consideramos que o trata- 
mento da hipertensão arterial é para toda a vida do paciente. Notamos ainda troca freqüente e desnecessária de diuréticos em um mesmo paciente encarecendo os gastos com medicação. Beta bloqueadores como o Propranolol, bem mais barato que outras drogas bloqueadoras adrenergéticas como o Metildopa, foram prescritos com muito menos freqüencia que esta. Reserpina foi raríssimamente prescrita.

A adoção de um tratamento padronizado e baseado na eficácia e preço das drogas anti-hipertensivas pode reduzir significativamente o aumento dos gastos decorrentes de medicar-se todos os pacientes hipertensos. Assim, se tivéssemos tratado os hipertensos leves (67 casos) com Hidroclorotiazida $50 \mathrm{mg} /$ dia, os hipertensos moderados (17 casos) com Hidroclorotiazida $50 \mathrm{mg} / \mathrm{dia}+$ Propranolol $120 \mathrm{mg} /$ dia (dose média) e os hipertensos graves (12 casos) com hidroclorotiazida $50 \mathrm{mg} / \mathrm{dia}+$ Propranolol $120 \mathrm{mg} /$ dia + Minipress $5 \mathrm{mg} /$ dia, o gasto anual total com os 96 casos seria de Cr $\$ 1.222 .820,00$, dando um gasto médio anual por paciente de $\operatorname{Cr} \$ 12.737,00$, pouco abaixo do gasto médio anual/paciente encontrado com o tratamento medicamentoso de apenas 60 casos (Cr\$ 14.110,00). Uma pequena mudança neste esquema: Higroton $50 \mathrm{mg} /$ dia para os hipertensos leves, Higroton + Aldomet $(750 \mathrm{mg} / \mathrm{dia})$ para os hipertensos moderados e Higroton $50 \mathrm{mg} / \mathrm{dia}$ + Aldomet $(750 \mathrm{mg} / \mathrm{dia})+$ Minipress $5 \mathrm{mg} /$ dia para os hipertensos graves mais do que dobraria o gasto médio anual por paciente que atingiria $\mathrm{Cr} \$$ $30.025,00$.

$O$ custo anual total por paciente, de US\$ 103.00 por nós encontrado é menor do que o descrito por Weinstein e Stanson 13 - US\$ 200.00 , assim dividido: US\$ 30.00 para as consultas médicas, US\$ 30.00 para os exames laboratoriais e US $\$ 140.00$ para a medicação, ou ainda o custo mencionado por Freis 2 de US\$ 500.00/ano sem discriminar suas partes componentes.

Vemos portanto, que os componentes do custo direto total anual por nós encontrado são inteiramente diversos dos de Weinstein e Stanson 12. Enquanto estes autores dão $70 \%$ dos custos como dependentes dos gastos com medicação, encontramos que, no nosso caso, $63,7 \%$ dos custos dependem dos custos das consultas e das intemações as quais são dependentes das complicações da doença.

A inexistência de um programa comunitário para detecção, avaliação, tratamento e controle do paciente hipertenso está ocasionando altos custos individuais e sociais devido a alta morbidade e mortalidade desta doença quando não tratada. Nas cinco principais capitais brasileiras as doenças do aparelho circulatório ocupam o primeiro lugar entre as causas de morte, sendo a $\mathrm{HA}$ a responsável direta ou indireta na grande maioria destas mortes por ser importanie fator de risco na doença isquêmica do coração e nas doenças cérebro-vasculares. HA está entre as três principais causas de invalidez temporária sendo responsável por 49.942 licenças concedidas em 1982 pelo Instituto Nacional de Previdência Social (INPS) com um período médio de duração do benefício de 268 dias. HA é também a principal causa de invalidez permanente (excetuando-se os acidentes de trabalho) tendo sido o motivo de 29.613 aposentadorias, concedidas pelo INPS em 19829. Estas aposentadorias ocorrem 12 anos mais cedo do que a média por outras causas.

Se considerarmos que cada paciente em benefício temporário ou permanente recebe um salário minimo (USS 36.21), o INPS estaria gastando por ano US\$ $14,467,198.00$ com novos benefícios temporários e US $\$ 12,867,440.00$ em novas aposentadorias. Considerando o custo direto hospitalar encontrado no presente trabalho de US\$ 103.00/ano e que, da população adulta brasileira (censo de 1980) $11,3 \%$ são hipertensos 7 , e ainda que apenas $30 \%$ da população de hipertensos adultos está diagnosticada (2,31 milhōes de hipertensos), o custo hospitalar anual para tratar estes pacientes alcançaria US\$. $237,930,000.00$. Se reduzirmos o nivel de $\mathbf{P A}$ considerado como critério diagnóstico de 160/95 $\mathrm{mmHg}$ conforme utilizado por Klein 7 para $140 / 90$ $\mathrm{mmHg}$, critério por nós utilizado, a prevalência da doença passaria a cerca de $30 \%$. Com isto a população de hipertensos adultos no Brasil passaria de 7,7 milhões para 20,44 milhões e o custo para tratar apenas os $30 \%$ já detectados $(6,13$ milhoes) elevar-se-ia para US\$ $631,390,000.00$ evidenciando as enormes conseqüências de uma simples alteração do critério diagnóstico da hipertensão arterial. Estes custos somar-se-iam aos custos da invalidez temporária e permanente acima apontados mostrando o quanto uma doença pode drenar dos recursos da sociedade, mesmo sem considerar a perda de produtividade conseqüente. Torna-se, portanto, prioritária uma ação eficaz para controle da $\mathrm{HA}$, de modo a evitar que os pacientes apresentem complicações e de modo a reduzir as graves repercussões individuais e sociais apontadas. Estas ações também carregam um custo, o qual é certamente menor do que o custo atual dependente em boa parte de uma ação ineficaz do nosso sistema de saúde e das condições sócio-econômicas de nossa população, entre outras causas.

O presente trabalho procura dar subsídio para que outros estudos semelhantes de minimização de custos, ou mais complexos de custo-benefício ou custo utilidade, se desenvolvam. A importância destes estudos é evidenciada pela definição de hipertensão arterial preconizada por Kaplan6: "Hipertensão arterial é a pressão arterial na qual os benefí. 
SOUZA E SILVA, N.A. de et al. Importância clínica dos custos diretos hospitalares em pacientes com hipertensão arterial em tratamento num hospital universitário, Rio de Janeiro, Brasil. Rev.Saúde públ., S. Paulo, 20: 293-302, 1986.

cios da ação menos os custos e riscos desta ação excedem os custos e riscos da inação menos os benefícios desta inação".

A minimização dos custos diretos hospitalares na avaliação e tratamento do paciente hipertenso, em nosso meio, considerando os fatos apontados, pode ser alcançada através das seguintes medidas:

1) Desenvolver programas que impliquem em melhor controle do paciente hipertenso com a finalidade de reduzir as complicações e, portanto, a útmanda na utilização do sistema de saúde pelo paciente, bem como reduzir o abandono do tratamento o qual, se por um lado ocasiona um aumento da demanda, por outro a reduz possibilitando melhor controle da doença e, portanto, menor número de complicações.

2) Padronizar a avaliação clínica e laboratorial inicial e seqüencial do hipertenso utilizando como exames complementares mínimos apenas: EAS, creatinina sérica, K+sérico e talvez ECG, valorizando-se os dados da anamnese e exame físico, para indicação ou não dos exames complementares.

3) Padronizar o tratamento anti-hipertensivo utilizando medicarnentos de baixo preço e eficácia já demonstrada: Hidroclorotiazida para os hipertensos leves, Hidroclorotiazida + reserpina ou beta bloqueador para os hipertensos moderados e Hidroclorotiazida + -reserpina ou beta bloqueador + Hidralazina ou Prasozin para os hipertensos graves.

4) Tratar o paciente hipertenso já diagnosticado mantendo-o em acompanhamento continuo e sob controle em unidades de saúde onde os custos indiretos pouco influenciem o custo das consultas e das internações.

SOUZA E SILVA, N.A. de et al. [ The clinical importance of direct hospital costs in patients with arterial hypertension under treatment in an university hospital, Rio de Janeiro State, Brazil ]. Rev. Saúde públ., S. Paulo, 20: 293-302. 1986.

ABSTRACT: The authors analysed retrospectively 503 adult patients $<20$ years old) representing 9.6\% of the population living in the XX Administrative Region of Rio de Janeiro and registered for treatment in the University Hospital. UFRJ in the year of 1978 (5,262 patients). Arterial blood pressure was recorded in 483 of these cases and $138(28.6 \%)$ were considered to have primary arterial hypertension ( $R P \geqslant 140 / 90 \mathrm{mmHg}$ ). The 96 cases initially analysed were studied for the determination of the direct hospital costs involved in the evaluation, follow-up and treatment of such cases. The initial diastolic BP was distributed as follows: 90 to $104 \mathrm{mmHg}-67$ cases $(69.8 \%) ; \quad 105$ to $114 \mathrm{mmHg}-17$ cases (17.7\%); $\geqslant 115 \mathrm{mmHg}-12$ cases $(12.5 \%)$. The mean follow-up period of this population was 653 days and at the last hospital visit only $1 / 3$ had their BP under control ( $\angle 140 / 90 \mathrm{mmHg}$ ). By $1982,68.7 \%$ had abandoned their treatment. The total annual direct hospital costs per patient were $\$ 102.48$ thus distributed: ambulatory consultations determined by the hypertension $\$ 33.44$; emergency consultations $\$ 2.33$; hospital admissions $\$ 29.92$; laboratory tests $\$ 10.45$; expenses with anti-hypertensive medications $\$ 26.34$. The disease morbidity determined the great majority of the hospital consultations and admissions $(64 \%$ of the costs). Disease morbidity also causes high indexes of temporary and permanent disability with major social costs. The analysis of the final costs determinants indicated the following measures for costs minimization: a) Development of programs within the health units with a view to better BP control and aiming at reducing the abandonment of treatment by the patients; b) Standardization of the laboratory evaluation and treatment, based on studies of cost-efficiency; c) The establishment of a hierarchy among the health units. The hypertensive patient must be treated in health units where indirect hospital costs do not much influence the final costs of hospital consultations and admissions.

UNITERMS: Hypertension, economics. Hospitals. Costs and cost analysis.

\section{REFERENNCIAS BIBLIOGRĀFICAS}

1. THE CANADIAN HYPERTENSION SOCIETY. Report of the consensus development conference on the management of mild hypertension in Canada. Toronto, 1984.

2. FREIS, E.D. Sounding board: should mild hypertension be treated? New Engl. J. Med., 307: 306-9, 1982.

3. HYPERTENSION DETECTION AND FOLLOW-UP PROGRAM COOPERATIVE GROUP. Five year findings of the hypertension detection and follow-up program. I - Reduction in mortality of persons with high blood pressure, including mild hypertension. J.Amer. med. Ass., $242: 2562$ - 71, 1979.
4. IZZO, J.L. Renovascular hypertension: diagnostic problems and therapeutic implications; Editorial. J. Amer. med. Ass., 249: 398-9, 1983.

5. JOINT National Committee on Detection, Evaluation and Treatment of High Blood Pressure; the 1984 report. Arch. intern. Med., $144: 1045-57$, 1984.

6. KAPLAN, N.M. Whom to treat: the dilema of mild hypertension. Amer.Heart J., 101: 867-70, 1951.

7. KLEIN, C.H. A hipertensão arterial em estratos geoeconômicos do Rio Grande do Sul. Rio de Janeiro, 1981. [Dissertação de Mestrado - Escola Nacional de Saúde Pública]. 
SOUZA E SILVA, N.A. de et al. Importância clínica dos custos diretos hospitalares em pacientes com hipertensão arterial em tratamento num hospital universitário, Rio de Janeiro, Brasil. Rev.Saúde públ., S. Paulo, 20: 293 -302, 1986.

8. MANAGEMENT COMMITTEE OF THE AUSTRA. LIAN THERAPEUTIC TRIAL IN MILD HYPERTENSION. The australian therapeutic trial in mild hypertension. Lancet, $1: 1261-7,1980$.

9. MINISTERRIO DA PREVIDĒNCIA E ASSISTÊNCIA SOCIAL. Instituto Nacional de Previdência Social. Secretaria de Serviços Previdenciários. A atuaçāo da perícia médica. Brasília, 1982.

10. SMITH, W.M. Treatment of mild hypertension: results of a ten year intervention trial. US Public Health Service Hospital Cooperative Study Group. Circ.Res., (suppl. 1) : 40-105, 1977.
11. VETERANS ADMINISTRATION COOPERATIVE STUDY GROUP ON ANTIHYPERTENSIVE AGENTS. Effects of treatment on morbidity in hypertension: results in patients with diastolic blood pressures averaging 115 trough $129 \mathrm{mmHg}$. J.Amer. med.Ass., 202: 116-22, 1967.

12. WEINSTEIN, M.C. \& STASON, W.B. Economic considerations in the management of mild hypertension. Ann.N.Y.Acad.Sci., $304: 424-40,1978$.

Recebido para publicação em 22/11/1985

Reapresentado em 21/05/1986

A provado para publicação em 02/06/1986 